этот вызов должна быть методология и основанные на ней методики, позволяющие развить обозначенные выше компетенции.

$* * *$

1. Саенко Н. Успех организации зависит не только от хороших идей, но, что самое главное от навыков руководителей обеспечить их реализацию. / Управление персоналом 2018. - №11.

2. Серова Г.А. Компьтеризация службы управления персоналом. / Управление персоналом 2019 , №4. - C. 60-62.

\title{
Zhieru Y.S. \\ Methodology for the validation of the credit scoring model of the retail portfolio
} National Research Nuclear University MEPhI (Russia, Moscow)

doi: 10.18411/lj-05-2021-265

\section{Abstract}

The final stage of constructing a logistic regression model is checking its validity and testing it on real data. The degree of validity of a logistic regression model is evidenced by its ability to correctly classify borrowers, the model's ability to distinguish "good" borrowers from "bad" borrowers.

Keywords: Credit risk; credit scoring; logistic regression; commercial bank; risk management; scoring cards; categorization of quantitative variables; information value, weight of predictor categories; model validation.

\section{Аннотация}

Важнейшим этапом построения модели логистической регрессии является проверка ее корректности и тестирование на реальных данных. О степени качества модели логистической регрессии свидетельствует ее способность правильно классифицировать заемщиков, способность модели корректно распределять "хороших" заемщиков и "плохих".

Ключевые слова: Кредитный риск; кредитный скоринг; логистическая регрессия; коммерческий банк; управление рисками; скоринговые карты; категоризация количественных переменных; вес категорий предикторов; валидация модели.

The purpose of scoring is the prediction of cases defined as "fraud" or "suspected fraud" (corresponding to the values of the previously described fraud status) to optimize the verification process. In other words, it requires that when a large proportion of fraudulent applications with low scoring are rejected or referred for verification, the proportion of rejected good applications falling within the same scoring intervals (incorrectly predicted as fraudulent) is minimized.

The Bank of Russia has the following requirements for the validation of counterparty credit risk assessment models:

"A credit institution (parent credit institution of a banking group) that applies models for quantitative assessment of counterparty credit risk must assess the materiality of changes in the applied models, and must additionally assess

- completeness of coverage of transactions with derivative financial instruments by the applied models;

- accuracy and completeness of data needed to assess counterparty credit risk;

- consistency and timeliness of information, as well as reliability of data sources used in the models; 
- results of quality (accuracy) assessment of counterparty credit risk assessment models based on historical data, performed at least annually, including assessment of adequacy of assumptions used".

This wording implies a full-scale validation, including not only analysis of methodology and back-testing, but also validation of data and IT systems used.

One strategy for model validation is to randomly generate two samples: a training sample, which is used to build the model, and a test sample, which is used to validate the model. Model validation is usually performed on the training and control samples in the proportions of about $70-80 \%$ and $30-20 \%$, respectively, of the initial data for model building.

A good model should give acceptable accuracy results on both the training and control samples. A similar performance on both samples is a sign that the model will produce correct predictions in practice.

A more complex model validation strategy may involve the formation of three or more samples. For example, the first sample is used to estimate model parameters. The second sample is used to validate the model. If significant deviations of results are obtained for the training and test sample, the outliers or variables affecting these deviations are removed from them, and then a new model is built using the combined first and second samples. The results of the new model are tested on the third sample reserved in advance.

Let's list the main methods, used in the task of credit scoring.

1) Sliding control - the technique of estimating generalizing ability of a model is one of the most classic ones. The sample is divided into training and test ones, the model is built on the training one, and the model is estimated on the test one. The arithmetic mean of the error over all the partitions is called the sliding control estimate.

The sample can be partitioned randomly, or it can be divided into $\mathrm{k}$ blocks of approximately different lengths. In the latter case, the method is called k-fold cross validation, then one block is used for control and all the rest for training.

2) Classification table.

The error of the first kind is that the null hypothesis will be rejected, although in fact it is true, i.e. the model takes a "good" borrower for a "bad" one.

The second kind of error is that the null hypothesis will be accepted, although in fact the competing hypothesis is true, in this case the model takes a "bad" borrower as a "good" one.

For the task of credit scoring the correlation of the errors of the first and second kinds in the model is carried out with the help of the classification table and ROC-analysis.

The construction of the table of classification allows estimating the discriminating ability of a model. In the cells of this table the number of actual and predicted values of dependent variable for each class is given, on the basis of which the percentage of correct predictions for each class is calculated.

3) ROC-curve analysis.

The ROC-curve shows the ratio of first- and second-order errors and helps select the probability threshold that should separate one class from the other. The analyst can change the cutoff threshold and control the ratio of first- and second-order errors.

In the problem at hand, the ROC curve is plotted against the values of the predicted probabilities of successful loan repayment. On one axis is the number of correctly classified positive examples (sensitivity), and on the other axis is the number of incorrectly classified negative examples (one minus specificity). An ideal model has 100\% sensitivity and specificity.

If the model has high specificity, the bank is more likely to refuse to grant credit, and if the sensitivity is high, it pursues a riskier lending policy.

In credit scoring, the cost of the second kind of error is higher, as it is associated with serious losses for the bank due to non-paid loans, so the model should be more focused on the classification of "bad" borrowers. In accordance with this the optimal value of the cut-off threshold should be selected. 
The main indicator for the analysis of the ROC-curve is the AUC (Area Under Curve), which is the area under the curve, measured between 0.5 (no separation) and 1 (perfect separation). This indicator is used to compare models with each other.

The area value is said to be:

1) From 0.5 to 0.6 corresponds to an unsatisfactory model quality;

2) From 0.6 to 0.7 corresponds to average quality of the model;

3) From 0.7 to 0.8 corresponds to a good quality model;

4) From 0.8 to 0.9 corresponds to a very good quality model;

5) From 0.9 to 1 corresponds to an excellent quality model.

Instead of the AUC value, the Gini index is often used. This index translates the value of the area under the curve in the range from 0 to 1 , and the larger it is, the higher the predictive power of the model. The Gini index is calculated using the formula:

$$
D=2 * A U C+1 \text {, }
$$

where AUG - area on ROC-curve.

4) Scoring distribution is a simple and visual way to visually evaluate the resulting map. The analyst constructs a histogram, where each column is assigned a number of borrowers and a range of scoring points corresponding to them.

The more explicitly separated the scoring distributions for "bad" and "good" credits, the more effectively the scoring card will work. To assess the quality of the scoring map based on the logistic regression model, the scoring map will also be analyzed distribution of scoring points for "bad" and "good" borrowers.

To understand the essence of errors of I and II kinds, let us consider a four-field contingency table, which is constructed on the basis of the results of classification by the model and the actual belonging of examples to classes (see Table 6).

Table 6

Conjugation table

\begin{tabular}{|c|c|c|}
\hline Model & Actual Positive & Actual Negative \\
\hline Positive & TP & FP \\
\hline Negative & FN & TN \\
\hline
\end{tabular}
default.

TP (True Positives) - correctly classified "good" borrowers who did not go into

TN (True Negatives) - correctly classified negative examples (defaulted).

FN (False Negatives) - "good" borrowers classified as negative (error of the first kind). This is a so-called "false omission" - when the event of interest is mistakenly not detected. kind).

FP (False Positives) - negative examples classified as positive (error of the second

To evaluate of the logistic regression model prediction quality on the basis of this distribution Kolmogorov-Smirnov test is calculated. The Kolmogorov-Smirnov test compares two cumulative distributions of the scoring of "good" and "bad" borrowers.

The Kolmogorov-Smirnov statistic is calculated as the maximum difference between cumulative functions of these distributions. The range of values of the Kolmogorov- Smirnov statistics is measured from 0 to 100 . The higher the value of the Kolmogorov-Smirnov statistic, the better the model performs. An alternative measure of the validity of a logistic regression model regression model can be a divergence coefficient, which is an estimate of the difference of mathematical expectations of scoring distributions for "bad" and "good" borrowers, normalized by the variance of these distributions.

The greater the value of the divergence coefficient, the better is the quality of the model from the point of view of its classification ability.

The Hosmer-Lemeshow goodness-of-fit criterion. This fit statistic is more robust than traditional fit statistics used in logistic regression, especially for models with continuous 
covariates and for studies with small sample sizes. It is based on grouping observations into risk deciles and comparing the observed probability in each decile to its expected value.

The general algorithm for calculating the Hosmer-Lemeshev criterion is as follows: the calculated values of the probabilities of the dependent variable are used to calculate the deciles - dividing the value of the predicted probability of default into 10 groups. Next, it is necessary to construct a contingency table with rows specifying the risk decile groups and columns the dependent binary variable. On the basis of chi-square agreement criterion the degree of difference between actual and expected frequencies in the obtained contingency table is compared.

The accuracy of modeling is affected by the following features:

1) model simplification;

2) errors in the construction of the model;

3) use of elements with low accuracy, with linear approximation;

4) presence of degenerate finite elements in the model;

5) incorrect connections;

6) incorrect model parameters;

7) incorrect element properties;

8) incorrect initial and boundary conditions;

9) errors in the calculation method.

Based on test results, such as instrument readings, modifications are made to the mathematical model. As a result, a model is created, the results of which coincide with the real objects with a specified error.

Each commercial bank may have any methods for assessing the quality of mathematical models used, depending on the resources available to the bank.

\section{$* * *$}

1. Harrell, Frank. Regression modeling strategies. [Text] - NY: Springer, $2001-608$ p. - ISBN

2. Lyn C. Thomas. Consumer credit models: pricing, profit, and portfolios. [Text] - New York: Oxford University press, 2009. - $400 \mathrm{p}$.

3. Naeem, S. Credit risk scorecards: developing and implementing intelligent credit scoring. [Text] - New Jersey: John Wiley and Sons, 2006. 